\title{
THE PRACTICAL VALUE OF THE FROG TEST IN THE DIAGNOSIS OF NORMAL AND PATHOLOGICAL PREGNANCY
}

\author{
BY \\ ERWIN RABAU AND ARYEH SZEINBERG \\ From the Government Hospital, Tel-Hashomer, Israel
}

(RECEIVED FOR PUBLICATION JULY 17, 1958)

The laboratory diagnosis of pregnancy, initiated by the discovery of the Aschheim-Zondek reaction, remained for a long time a comparatively seldom used procedure, performed only on selected cases. The original method and its later modifications (the Friedman test, the quick hyperaemia test) need comparatively expensive test animals. Moreover, the performance of the test and reading of the results is by no means always easy and requires a highly skilled laboratory staff. One of the simpler procedures, the female Xenopus Lewis frog test, although much advocated for some time, was found to lack sensitivity and the animals were difficult to breed and maintain outside their native land. The various chemical procedures proposed lacked both sensitivity and specificity and therefore have been disappointing. A new era in this field was initiated by GalliMainini, who described the use of several species of South American male frogs for the diagnosis of pregnancy (Galli-Mainini, 1948). The procedure became universally known as the "frog test" and very favourable reports about its use were soon published in the medical press of many countries (Wiltberger and Miller, 1948; Haines, 1948 ; Hinglais and Hinglais, 1948 ; Nobili, 1948 ; Rabau and Szejnberg, 1949). The following is a summary of experience with this procedure during nearly 10 years of its routine use.

In nature the male frogs and toads release spermatozoa after a prolonged contact with the female, which stimulates the secretion of the gonadotrophic hormone from the hypophysis. A similar effect may be obtained by injecting human chorionic gonadotrophin excreted in urine during pregnancy, or in cases of hormone-secreting neoplasms (chorionic epithelioma, hydatid mole).

\section{Test Animals}

The suitability of the Batrachia for this procedure seems to be universal. Bhaduri in 1951 listed from the literature 34 species of frogs and toads belonging to 11 genera and eight families responsive to the injection of human chorionic gonadotrophin (Bhaduri, 1951). The three species indigenous to Israel (belonging to three different families, namely, the toad Bufo viridis, the river frog Rana ridibunda, and the tree frog Hyla arborea) were found suitable for the reaction (Szejnberg and Rabau, 1950). The care of the animals is exceedingly simple. In large laboratories where hundreds of frogs are used they may be kept in big tanks or aquaria with little water. In small laboratories or in a doctor's surgery any small glass jar placed in the shade may be used. The only care required is the daily change of water. Occasionally a fatal epidemic of the so-called "red leg disease" may spread in aquaria, but it may be overcome by sprinkling the aquaria with sulphadiazine or adding penicillin-streptomycin solution to the water, and promptly removing dead animals. In small laboratories where each test animal is kept in a separate container no danger of an epidemic exists.

The animals in captivity do not show much inclination for food (live worms) and sometimes have to be forced to swallow it. On the other hand, they seem to withstand very well prolonged periods of hunger extending even to several months. Although they become considerably emaciated during this period, they regain weight very easily when ultimately released. The usual procedure here is to use the animals for two or three months without feeding and then to release them.

The animals may be used repeatedly at an interval of at least seven days between consecutive injections, provided a control examination of the animals' urine is always made before the injection. On a few occasions spermatozoa were found in the urine of animals even a week after a previous positive response. Although the number of such cases was very small, they warranted a careful examination of each animal before use.

\section{Technique}

The urine $(1 \mathrm{ml} / / 10 \mathrm{~g}$. weight of animal) is injected subcutaneously into the dorsal lymph sac of the test animal. Before the injection and two hours after it a specimen of the animal's urine is procured from the cloaca with a capillary pipette and observed under the 
microscope. If negative, the examination is repeated after four hours.

Occasionally the urine is toxic to the animals as a result of drugs taken by the patients or to some unknown cause. This difficulty may be overcome in two ways: (1) By injecting a smaller volume of urine. This may be sufficient to give a positive result. If, however, the test is negative with the smaller volume, then the test should be repeated with the full amount of detoxified urine. (2) Of the various methods for detoxification, the following was found to be the most suitable for routine use : to $5 \mathrm{ml}$. of urine $20 \mathrm{ml}$. of ice-cold acetone is added, mixed, and left in the refrigerator for two hours. After centrifuging the supernatant is discarded, the precipitate washed once with cold acetone, and centrifuged again. The washed precipitate is left in the tube for $30 \mathrm{~min}$. at room temperature in order to evaporate the traces of acetone, and resuspended in $5 \mathrm{ml}$. water. Alternative efficient methods of detoxification which are quicker, requiring only 30 to 60 minutes for completion, but are technically somewhat more complicated were described by Scott (Scott, 1940 ; Brody, 1949 ; Cutler, 1949) (absorption from acidified urine with kaolin and elution with alkali) and by Robbins (dialysis of urine against running tap water) (Robbins, 1951).

\section{Sensitivity of the Animals}

The sensitivity of the animals was tested by injection of saline dilutions of international standard human chorionic gonadotrophin and calculating the ED50. A considerable seasonal variation in the sensitivity of the animals was observed. The Bufo viridis was about twice as sensitive during the winter season (time of growth and reproductive activity) than in the summer months. In winter 3 international human chorionic gonadotrophin units were sufficient to cause a positive response in $50 \%$ of the injected animals, while in summer about 6 units were required to cause $50 \%$ positive responses. The sensitivity changes in Rana ridibunda differed. This animal was more sensitive in summer (ED50 2.5 international units) than in winter (3.5 international units). The tree frogs (Hyla arborea) were used only during the winter and spring seasons, as they did not survive the summer in captivity. These small frogs weighed on the average 4-5 g. and their sensitivity range was about 0.6 to 0.8 international units human chorionic gonadotrophin.

The sensitivity of the animals did not change significantly after being used several times. Taking into account the individual and seasonal variations of sensitivity, no test was reported negative unless three animals were injected (preferably belonging to the three different species) and found negative.

\section{Quantitative Procedure}

For the quantitative estimation of the hormonal content two-fold dilutions of urine in water were prepared and $1 \mathrm{ml}$. of each dilution injected into the test animals. The maximal dilution giving a positive response was noted and the amount of the hormone in urine calculated as follows:

Dilution of urine $\times 1,000$ (frog units/litre).

Frog units/litre $\times$ mean sensitivity of the test animals (different for different seasons and animals) human chorionic gonadotrophin international units/ litre.

\section{Results \\ Diagnosis of Pregnancy. - About 7,000} pregnancy tests have been performed during the period covered by this summary. The accuracy of the test depended on the length of amenorrhoea. The earliest examinations were performed four days after the date of the missed period. In some cases positive results have been obtained at such an early date, but on many occasions the test was negative to become positive a week later. The incidence of the positive results rose with each consecutive day of amenorrhoea, and with urines examined 12 days after the missed period or later no false negative results were found in our experience.

No false positive results were encountered when the test was performed with Bufo viridis or Hyla arborea. Rana ridibunda, on the other hand, was less reliable. On very rare occasions during the summer months only, a few spermatozoa could be detected in cases which later proved to be negative. These false positive results were probably caused by unspecific release of a few spermatozoa, as four or five additional Rana frogs injected with the same urine gave a uniformly negative result. Consequently, when during the summer months a very weak positive reaction was encountered in Rana ridibunda and additional animals injected were negative, the test was reported as inconclusive and an additional specimen of urine was requested for examination seven days later. Such cases were very rare, and with the above precautions no false positive results were reported by us during the entire period.

Value of Quantitative Test in Pathological Pregnancies.-The quantitative test has been used for the diagnosis of missed abortions as well as in the follow up of cases of habitual abortion. As a preliminary step for these investigations excretion levels of chorionic gonadotrophin in 200 normal pregnancies have been followed. It was found that individual variations in the range of excretion were very wide, but by calculation of the mean values for each gestation period, a hypothetical "normal curve" of chorionic gonadotrophin excretion could be drawn. This "normal curve" is characterized by a peak excretion during the 60-110th day of amenorrhoea. 


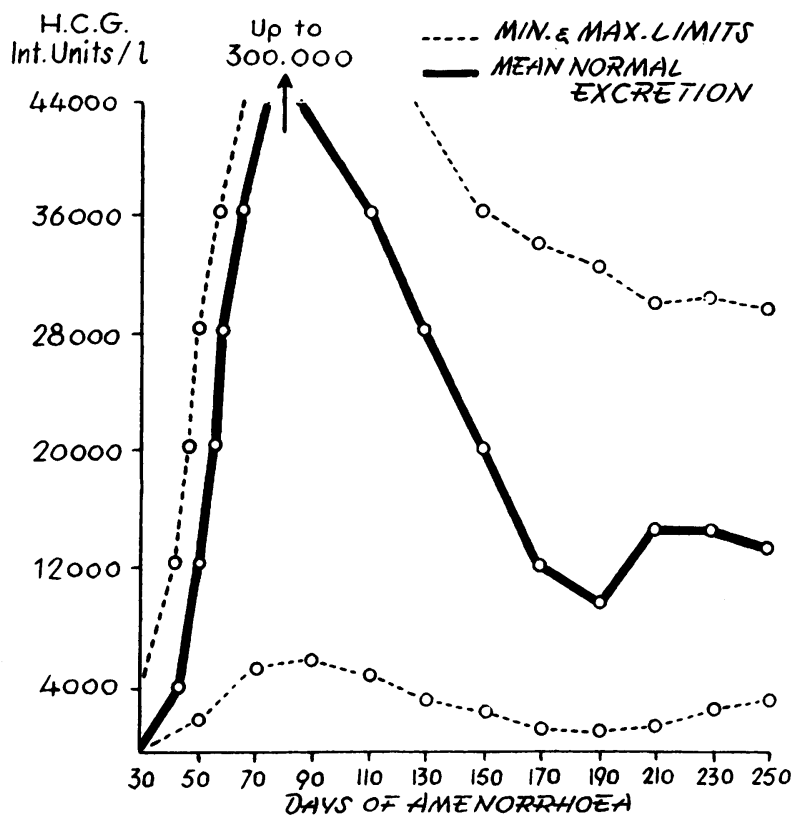

FIG. 1.-Excretion of chorionic gonadotrophin in normal pregnancies. for its decrease below the sensitivity level of the test animals (Fig. 2).

Up to now about 500 cases of suspected missed abortion have been investigated by the quantitative method, and the following conclusions can be drawn.

(1) A single estimation of the gonadotrophin level cannot serve as a basis for the diagnosis of foetal death unless the frog test is negative. When the examination was performed a few days after the suspected foetal death the hormonal level could be still very high (especially during the "peak period " of the hormonal excretion). On the other hand, the hormone titre could be low in cases when the foetus was still alive and normally developing, particularly during the fifth to sixth month of pregnancy, but also in some cases during the first trimester.

(2) Only repeated quantitative examinations performed at about one week's intervals can be of diagnostic value in cases of missed abortions. If a rise in the hormonal level or even maintenance of a "plateau" is detected on two consecutive

During this peak period most of the subjects excrete more than 32,000 international units chorionic gonadotrophin. After this period the levels usually drop, and during the fifth to sixth month of pregnancy very low values are encountered, approaching in some cases the limit of sensitivity of the test animals. Later the "mean value" rises again although to much lower levels than during the peak period (Fig. 1).

The second preliminary step consisted of investigating the rate of disappearance of chorionic gonadotrophin from urine after interruption of pregnancy. For this purpose the levels of the hormone were estimated before and after curettage. The results presented in Fig. 2 show that the speed of disappearance of the hormone from urine was related to the initial level before the intervention. When it was low, the urine had already become negative two or three days after the interruption of the pregnancy, while in cases with high initial levels even 14 days were sometimes required<smiles>[CH]=[Co]</smiles>

Int. Units/l.

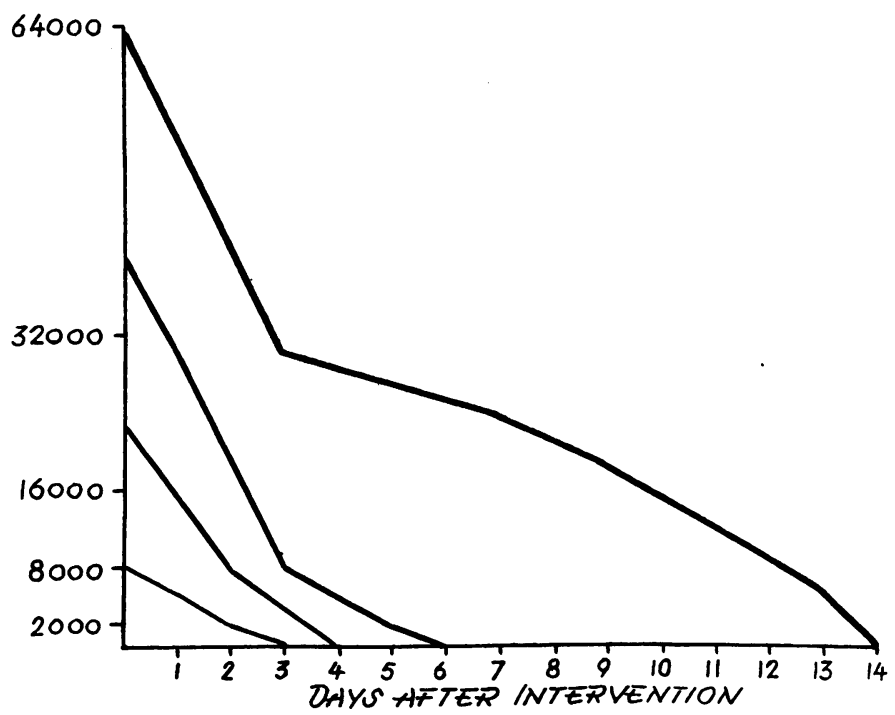

FIG. 2.-Changes in the chorionic gonadotrophin level in urine after curettage. examinations, the diagnosis of foetal death may be discarded with certainty. When, on the other hand, a decline in the level is observed after uterine bleeding or other unfavourable clinical signs (disproportion between the gestation period and the size of the uterus) a correlation with the 
normal trend of the "mean normal excretion curve" should be made and the case supervised carefully by repeated examinations until a final conclusion can be reached.

Follow-up in Cases of Habitual Abortion.The quantitative test has been performed at fortnightly intervals in about 100 cases of habitual abortion (cases with at least two previous spontaneous abortions).

(1) The chorionic gonadotrophin level in most cases of habitual abortion was within normal limits, the mean levels not being significantly lower than the mean normal values.

(2) The quantitation of the hormone had no prognostic value for the normal continuation of pregnancy. In some cases a finding of high hormonal excretion was followed a few days later by foetal death, while in other cases the pregnancy terminated well despite prolonged very low levels of the hormone and initial bleeding.

(3) While of no prognostic value, the test was very useful for the diagnosis of the foetal condition when performed regularly at two-week intervals. In most cases it eliminated the necessity for repeated gynaecological examinations, which in cases of habitual abortion were usually not easily agreed to by the patients and caused them unnecessary psychological stress. These patients often blame the doctor if foetal death occurs a short time after the physical examination. By these repeated tests foetal death can be relatively quickly diagnosed and the dead foetus removed, thus preventing unnecessary confinement to bed and the danger of fatal haemorrhage due to fibrinolysis.

(4) Some of the patients with a history of habitual abortion were given stilboestrol or progestine, but no medication was prescribed to the other patients. These drugs did not appear to affect the level of chorionic gonadotrophin excretion even when doses up to $100 \mathrm{mg}$. daily were given.

\section{Summary}

The "frog test" described by Galli-Mainini is a very useful procedure for the diagnosis of pregnancy. It is characterized by technical simplicity and a very high degree of accuracy.

Periodical hormonal estimations by this procedure are recommended during pregnancy in cases of habitual abortion and in cases of suspected missed abortions for prevention of unnecessary confinement to bed and of dangerous haemorrhagic complications due to fibrinolysis.

\section{REFERENCES}

Bhaduri, J. L. (1951). Proc. 38th Indian Sci. Congr., Vol. 2, p. 171. Brody, H. (1949). Amer. J. Obstet. Gynec., 57, 581.

Cutler, J. N. (1949). J. Lab. clin. Med., 34, 554.

Galli-Mainini, C. (1948). J. Amer. med. Ass., 138, 121. Haines, M. (1948). Lancet, $2,923$.

Hinglais, H., and Hinglais, M. (1948). C.R. Soc. Biol. (Paris), 142, 460 .

Nobili, L. (1948). Riv. ital. Ginec., 31, 352.

Rabau, E., and Szejnberg, L. (1949). Harefuah, 37, 15.

Robbins, S. L. (1951). J. clin. Endocr., 11, 213.

Robbins, S. L. (1951). J. clin. Endocr., 11, 213.

Scott, L. D. (1940). Brit. J. exp. Path., 21, 320. Wiltberger, P. B., and Miller, D. F. (1948). Science, 107, 198. 